\title{
7
}

\section{Estructuras y procesos para el gobierno de Tecnologías de la Información}

Diego Cordero Guzmán, Andrea Mory Alvarado.

Recibido: enero 2017

Aprobado: mayo 2017 


\title{
Estructuras y Procesos para el Gobierno de Tecnologías de la Información Structures and Processes for the Information Technologies Governance
}

\author{
Diego Marcelo Cordero Guzmán ${ }^{(1)}$ Andrea Vanessa Mory Alvarado ${ }^{(2)}$ \\ Unidad Académica de Tecnologías de la Información y la Comunicación ${ }^{(1)(2)}$ \\ Universidad Católica de Cuenca (UCACUE) \\ Cuenca-Ecuador \\ dcordero@ucacue.edu.ec ${ }^{(1)}$ \\ amorya@ucacue.edu.ec ${ }^{(2)}$
}

\begin{abstract}
Resumen
La investigación apoya a la generación de conocimiento para el Gobierno de las Tecnologías de la Información (TI), al interior de la organización. Para este cometido se propone un modelo para Gobierno de TI, el mismo que está formado por tres constructos o variables: estructuras, procesos y madurez del Gobierno de TI. El presente trabajo inicia con el análisis de la literatura, sección en donde se identifican las variables estructuras y procesos y se plantean las preguntas de investigación. A continuación, se proponen las hipótesis y el modelo de investigación. Se adjunta la revisión general de modelos de ecuaciones estructurales, estadístico que es utilizado para medida y prueba del modelo, el mismo que es sometido a instancia de prueba en dos universidades cofinanciadas de la Zona 6 de la República del Ecuador: la Universidad Católica de Cuenca (UCACUE) y la Universidad Politécnica Salesiana (UPS). Se exponen los resultados luego de haber aplicado los instrumentos al tamaño muestral seleccionado. A la vez la consistencia del modelo se lo valida a través de ecuaciones estructurales, para ello se usa el producto de software Smart PLS 3.1.9.; al final se levantan las conclusiones del estudio y se elaboran las sugerencias para futuras investigaciones.
\end{abstract}

Palabras Claves: Estructuras, procesos, madurez del gobierno de TI

\begin{abstract}
The research supports the generation of government knowledge for the Technological Information (TI), within the organization. For this purpose, a model for IT Governance is proposed, the same one that is formed by three constructs or variables: structures, processes, and maturity of the Government of TI. The present work initiates with the analysis of the literature, section where the variables structures and processes are identified and investigation questions appear. The following hypotheses and research models of investigation are proposed. Enclosed is the general review of structural equation models, which is statistically used for measuring and model testing, the same one that was submitted to test in two universities co-financed of Zone 6 of the Republic of Ecuador: The Catholic University of Cuenca (UCACUE) and the Salesian Polytechnic University (UPS). The results were then exhibited after having applied the instruments to the selected sample size. Simultaneously, the consistency of the model is validated through structural equations, using the software product Smart PLS 3.1.9.; in the conclusions of the studies questions and suggestions were raised for future research and elaboration.
\end{abstract}

Keywords: Structures, processes, maturity of IT governance 


\section{Introducción}

Para Cordero [1] en el Ecuador, para un considerable número instituciones de educación superior no existe el conocimiento adecuado sobre el marco de trabajo, que permita gobernar las Tecnologías de la Información, de modo que éstas no sean simples instrumentos tácticos u operativos, sino más bien se conviertan en herramientas estratégicas; por tanto, existe un problema latente al no saber ¿cómo gobernar de manera adecuada las tecnologías de la información (TI)?

El trabajo aporta con un modelo empírico para Gobierno de Tecnologías de la Información, que toma parte de la definición de Grembergen \& De Haes [2], en el sentido de que los procesos y las estructuras organizacionales habilitan la madurez del Gobierno de TI. De hecho, estas variables son los constructos básicos a partir de los cuales se constituye el Gobierno de TI [2].

Con las tres variables identificadas se plantean dos hipótesis, a ser demostradas en la investigación, así: H1, "Las estructuras influyen de manera positiva sobre la madurez del Gobierno de Tecnologías de la Información"; H2, "Los procesos influyen de manera positiva sobre la madurez del Gobierno de Tecnologías de la Información". Las hipótesis identifican la relación causal entre variables, bases para el modelo propuesto. El modelamiento, se desarrolla con ecuaciones estructurales de manera concreta con la técnica PLS (Partial Least Square), o mínimos cuadrados parciales.

El modelo se prueba con los datos levantados en dos universidades de la categoría cofinanciadas que pertenecen a la Zona 6 de la República del Ecuador, éstas son: la Universidad Católica de Cuenca (UCACUE) en donde se aplicaron 154 encuestas y la Universidad Politécnica Salesiana en Ecuador con 99 instrumentos ejecutados. De manera previa el instrumento fue validado a través de prueba expertos con la participación de informantes calificados, con conocimiento sobre gestión y Gobierno de TI.

Los datos se analizan a través de estadísticos de ecuaciones estructurales con la herramienta para modelamiento SMART PLS 3.1.9. Para al final levantar las conclusiones del estudio y recomendaciones sobre futuras investigaciones.

\section{Materiales y Métodos \\ 2.1. Marco Teórico}

Existen marcos de referencia y buenas prácticas que han sido generadas para una correcta gestión o gobernanza de TI, como el caso de COBIT 5, ITIL Val
IT Risk IT, TOGAF, entre otros. Más éstos han sido proporcionados para medioambientes organizacionales de países, en donde los niveles de madurez son superiores frente a un entorno de países que están en vías de desarrollo. No obstante, de ello muchas organizaciones han adoptado y adoptan las prácticas mencionadas. Pero también existe la oportunidad de generar o desarrollar, marcos de trabajo propios [1].

Para Kingsford et al. [3]; Brown y Grant [4], el entorno cultural de las regiones genera influencia sobre el Gobierno de TI. Por ello Zhong et al. [5], Nfuka and Rusu [6], indican que es recomendable particularizar los marcos de trabajo de Gobierno de TI para cada región, esto debido a que la mayoría de mejores prácticas para Gobierno de TI, han sido desarrollados para el entorno Anglosajón. Al mismo tiempo que es regionalizado un marco de trabajo, puede ser caracterizado para cada tipo de organización [7], [8]. Por tanto, es factible particularizar el marco de trabajo del Gobierno de TI para un entorno organizacional universitario y dentro de una región.

Dada la naturaleza del Gobierno de TI, a éste se lo concibe como parte del Gobierno Corporativo u Organizacional [9], sujeto a las mismas conveniencias y restricciones. El propósito del Gobierno de TI es descrito por el Instituto de Gobernanza de TI, [10], como: habilitar del alineamiento entre TI y la organización; apoyo de TI para explotar las oportunidades y maximizar los beneficios; administrar los recursos de TI de manera responsable; administrar los riesgos ligados a TI de manera responsable.

De acuerdo a la definición de Grembergen \& De Haes [2] y Peterson [11], el Gobierno de TI debe ser desarrollado a lo largo de una combinación de: estructuras, procesos y mecanismos relacionales. Sin embargo, la presente investigación asume dos de las tres variables, consideradas básicas para un buen Gobierno: las estructuras y los procesos de la organización; los mecanismos relacionales son opción para una futura investigación. A continuación, se explota el marco referencial de estas variables.

\section{Estructuras del Gobierno de TI}

Tiene que ver con unidades organizacionales, roles y responsabilidades para toma de decisiones de TI [12]. De acuerdo a De Haes \& Van Grembergen [13], Luftman \& Brier [14], las estructuras para implementar el Gobierno de TI son: estructura organizacional de TI; roles y responsabilidades de TI; el Comité Directivo de TI; el Comité Estratégico de TI; CIO (Oficial en jefatura de Sistemas), desempeña un papel de liderazgo en la 
promoción de TI [15] en el Comité Estratégico; CIO reportando al CEO (administrador máximo de la organización). Estas seis estructuras, se consideran para el dimensionamiento de la variable. En esta parte de la teoría se plantea la pregunta de investigación: ¿Cómo influyen las estructuras en el Gobierno de Tecnologías de la Información?

\section{Procesos del Gobierno de TI}

Implican las decisiones estratégicas y monitoreo de TI, de acuerdo a varios autores son: la planificación estratégica de tecnologías de información [16]; el tablero de comandos balanceado (BSC) [17], Parisa, Lazar y Shengnan [18]; los acuerdos de nivel de servicio (SLA), [19]; la información económica (Gestión de Cartera), [20]; las mejores prácticas como COBIT e ITIL. Sobre esta parte teórica se plantea la pregunta de investigación: ¿Cómo influyen los procesos en el Gobierno de Tecnologías de la Información?

\section{Madurez del Gobierno de TI}

El IT Governance Institute (ITGI), define 6 niveles de madurez para el Gobierno de TI, que van de desde 0 a 5 [21], como se especificada en la Tabla 1.

Tabla 1. Modelo de madurez del gobierno de TI.

\begin{tabular}{|c|l|l|}
\hline Nivel & \multicolumn{1}{|c|}{ Nombre } & \multicolumn{1}{c|}{ Descripción } \\
\hline 0 & No existe & No se aplican en lo absoluto. \\
\hline 1 & Inicial & $\begin{array}{l}\text { Son ah-hoc y } \\
\text { desorganizados. }\end{array}$ \\
\hline 2 & Repetible & Siguen un patrón regular. \\
\hline 3 & Definido & $\begin{array}{l}\text { Está implementado, } \\
\text { estandarizado y } \\
\text { documentado. }\end{array}$ \\
\hline 4 & Administrado & $\begin{array}{l}\text { Forma parte de la } \\
\text { organización se } \\
\text { monitorea y mide. }\end{array}$ \\
\hline 5 & Optimizado & Nivel de mejor práctica. \\
\hline
\end{tabular}

Fuente: Henderson, Venkatraman, \& Oldach [21]

\subsection{Metodología}

El problema de investigación está centrado en diseñar el Modelo para Gobierno de Tecnologías de la Información, para el sector universitario de la región, que relacione las variables: estructuras, procesos y madurez del Gobierno de TI.

\section{Formulación del Problema}

En la Tablas constan las preguntas de investigación que surgieron de la revisión de la literatura y las hipótesis planteadas.

Tabla 2. Interrogantes derivadas vs hipótesis

\begin{tabular}{|c|c|}
\hline Interrogantes & Hipótesis \\
\hline $\begin{array}{lrr}\text { ¿Cómo influyen las } \\
\text { estructuras en } & \text { el } \\
\text { Gobierno } & & \text { de } \\
\text { Tecnologías de } & \text { la } \\
\text { Información? } & & \end{array}$ & $\begin{array}{l}\text { Hipótesis } 1 \text { (H1): } \\
\text { Las estructuras influyen de } \\
\text { manera positiva sobre la } \\
\text { madurez del Gobierno de } \\
\text { Tecnologías de la } \\
\text { Información. }\end{array}$ \\
\hline $\begin{array}{lr}\text { ¿Cómo influyen } & \text { los } \\
\text { procesos en } & \text { el } \\
\text { Gobierno } & \text { de } \\
\text { Tecnologías de la } \\
\text { Información? }\end{array}$ & $\begin{array}{l}\text { Hipótesis } 2 \text { (H2): } \\
\text { Los procesos influyen de } \\
\text { manera positiva sobre la } \\
\text { madurez del Gobierno de } \\
\text { Tecnologías de la } \\
\text { Información. }\end{array}$ \\
\hline
\end{tabular}

Fuente: autores

\section{Modelos de ecuaciones estructurales (MEE)}

El modelo de la investigación es resuelto con ecuaciones estructurales, las mismas que son de utilidad relevante en el análisis multivariante [22]. Pueden ser aplicadas con gran factibilidad en procesos de administración en la organización, por tanto también en administrar la tecnología [23]. A través de los MEE es posible valorar $[24$, p. 6$]$ :

- El modelo de medida (outer model), que implica determinar las cargas factoriales de las variables observables (indicadores) en relación a sus variables latentes (constructos).

- El modelo estructural (inner model), formado por las relaciones de tipo causal que han sido definidas como hipótesis.

La figura 1, representa los dos componentes de los MEE: el modelo de medida u outer model y modelo estructural o inner model. 
Figura 1. Componentes del MEE

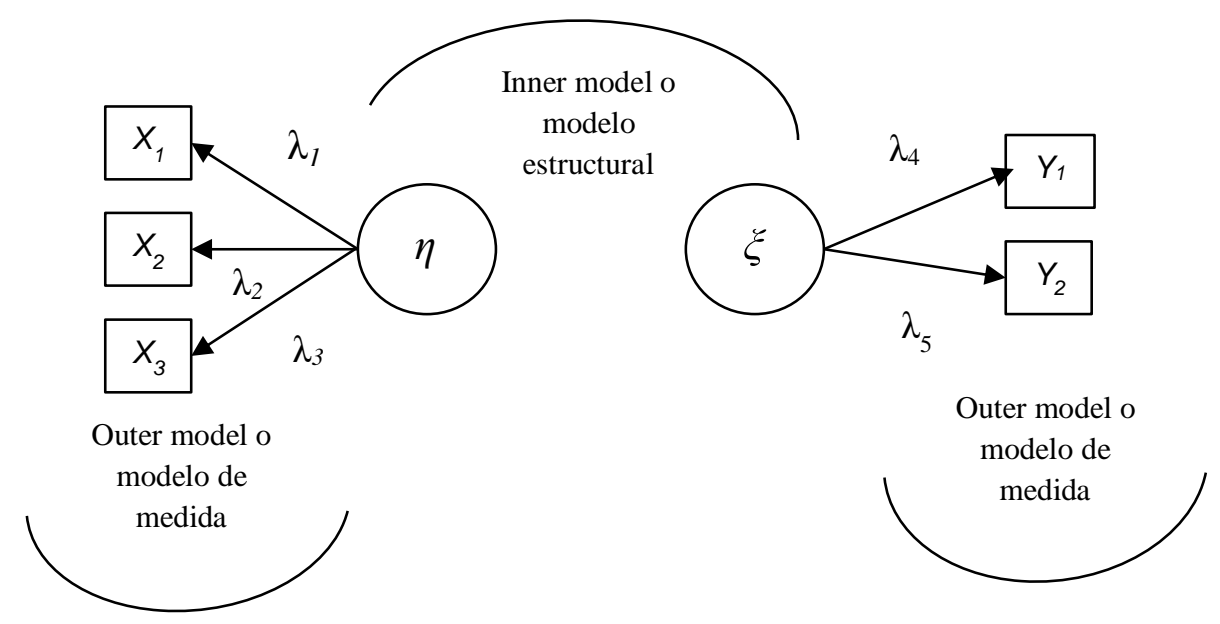

Fuente: Caballero Domínguez [25, p. 60]

\subsection{Modelo propuesto}

Con las hipótesis planteadas, se propone el modelo especificado en la figura 2, en donde constan tres constructos:
1. ESTGTI. Estructuras del Gobierno de Tecnologías de la Información.

2. PRCGTI. Procesos del Gobierno de Tecnologías de la Información.

3. MGTI. Madurez del Gobierno Tecnologías de la Información

Figura 2. Modelo para Gobierno de TI

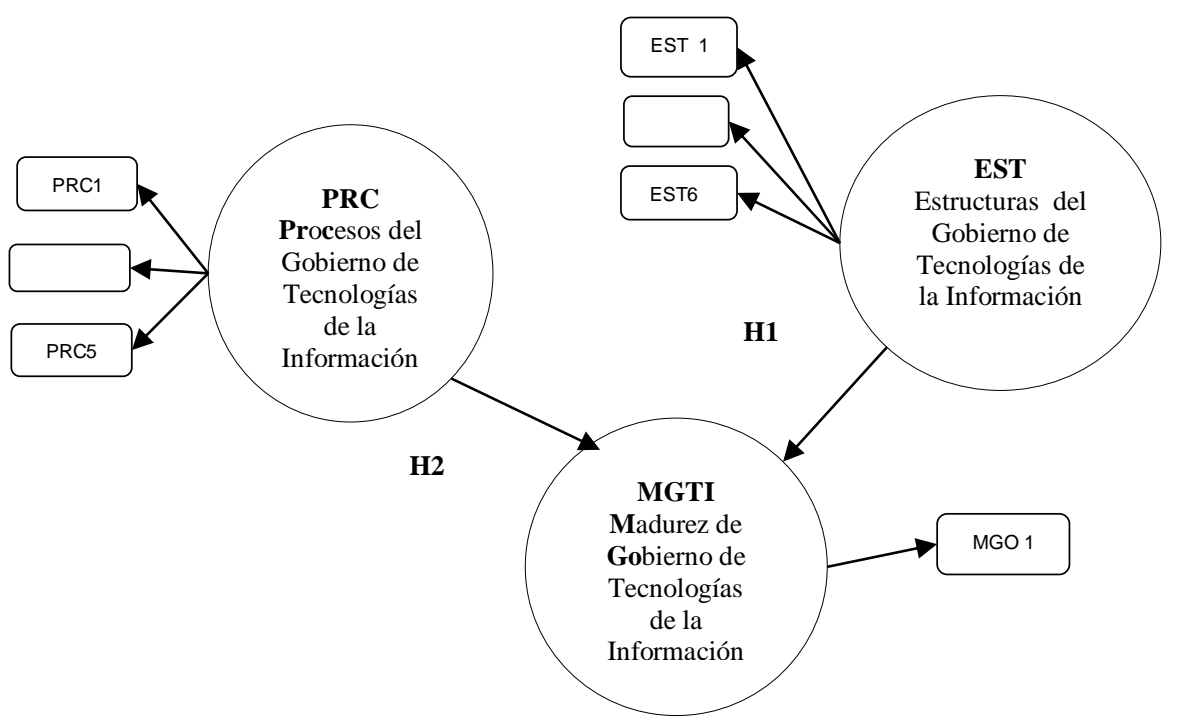




\section{Caracterización de la Investigación}

La investigación es:

- Exploratoria. Por los pocos estudios previos sobre el Gobierno de TI en entornos organizacionales en la región y más aún en la universidad.

- Explicativa. Porque, busca dar cuenta del Gobierno de Tecnología en la organización.

- Correlacional. Dado que el objetivo del estudio es encontrar relaciones entre las variables analizadas.

\section{Contexto de la aplicación del modelo}

La Universidad Católica de Cuenca (UCACUE), de categoría cofinanciada fue creada el 7 de septiembre de 1970. Oferta carreras de tercer nivel en las ramas de las ciencias sociales, ingeniería, ciencias de la salud, ciencias empresariales y económicas. Se caracteriza por brindar una educación de calidad y cristiana; e imparte maestrías de cuarto nivel, debidamente aprobadas por el CES (Consejo de Educación Superior).

Su matriz está en la ciudad de Cuenca, provincia del Azuay. Cuenta con las Sedes Universitarias en la ciudad de Macas, provincia de Morona Santiago desde 1983; en la ciudad de Azogues, provincia del Cañar desde 1980; y en esa misma provincia las extensiones de San Pablo de La Troncal y Cañar desde 1990, creaciones que fueron respuesta a las necesidades del pueblo, el desarrollo integral de la región y a la presencia masiva de las juventudes estudiosas.

El 8 de mayo de 1984 se crea la Modalidad de Estudios a Distancia o No Presenciales con su Matriz en Cuenca y centros de Apoyo en Quito, en Tena, en Zamora y, Méndez, ampliando la tarea universitaria en la nación, el continente y el mundo, en la doble vertiente de presencial y no presencial.

A la fecha cuenta con 27 carreras (diseñadas y rediseñadas) de acuerdo a la exigencia del mundo contemporáneo y acorde con los lineamientos de los órganos de control de la educación superior en el
Ecuador; y, como institutos Anexos: 2 Centros Infantiles, 6 Escuelas Superiores; y, 4 Colegios Universitarios.

Cuenta con una planta docente universitaria de 800 profesores con perfiles de Maestría, con la respectiva pertinencia. El número de estudiantes, en la universidad ( $\sin$ contar con planteles anexo), en la actualidad bordea los 11,900.

La Universidad Politécnica Salesiana (UPS), creada con Decreto Ejecutivo del Gobierno del Ecuador, el cuatro de agosto de 1994, da inicio a sus actividades académicas en octubre del mismo año. Tiene tres sedes: la principal ubicada en la ciudad de Cuenca y las restantes en las ciudades de Quito y Guayaquil.

El nivel directivo está estructurado por el Rector, el Vicerrector Académico General, el Vicerrector Docente y los Vicerrectores de las Sedes. Los cuerpos colegiados rectores están integrados por el Consejo Superior, el Consejo Económico y Financiero, el Consejo Académico.

Cuenta en la actualidad con 1,000 docentes y una media de 17,200 estudiantes, con una oferta académica distribuida en carreras de grado y posgrado, en las áreas de: la ingeniería, veterinaria, las ciencias sociales, educativas. Cada carrera dispone de sus respectivos órganos colegiados para sus procesos administrativos y de ejecución de cátedra.

\section{Población y muestra}

Para determinar la muestra de ¿a quiénes? aplicar los instrumentos, se toma como referencia las características del modelo propuesto (12 indicadores, 3 constructos, 2 el número de constructos que apuntan a una variable dependiente). De acuerdo a la "modelización PLS", el tamaño muestral para el modelo es de $10 * 2=20$. Es decir, que son suficientes 20 encuestas para probar el modelo. Sin embargo, se aplicaron 253, para dar mayor consistencia a los resultados. 
Tabla 3. Instrumento para captura de datos

\begin{tabular}{|c|c|c|}
\hline ID & Ítems & Escala \\
\hline \multicolumn{3}{|c|}{ 1. Estructuras del Gobierno de Tecnologías de la Información } \\
\hline EST1 & ¿La toma de decisiones sobre tecnologías de la información? & 01212345 \\
\hline EST2 & ¿Roles y responsabilidades sobre tecnologías de la información? & 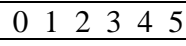 \\
\hline EST3 & ¿El Comité Estratégico de Tecnologías de la Información? & 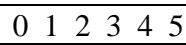 \\
\hline EST4 & ¿El Comité Directivo de Tecnologías de la Información? & 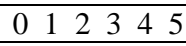 \\
\hline EST5 & ¿El Director (CIO, jefe, gerente) de TI, forma parte del Comité Estratégico de TI? & $\begin{array}{llllll}0 & 1 & 2 & 3 & 4 & 5\end{array}$ \\
\hline EST6 & ¿El Director (CIO, jefe, gerente) de Tecnologías de la Información, reporta sus actividades? & 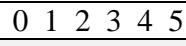 \\
\hline \multicolumn{3}{|c|}{ 2. Procesos del Gobierno de Tecnologías de la Información } \\
\hline PRC1 & ¿La planificación estratégica de tecnologías de la información? & 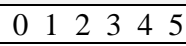 \\
\hline PRC2 & ¿Indicadores y medidas sobre las tecnologías de la información? & 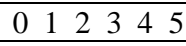 \\
\hline PRC3 & ¿Acuerdos sobre el nivel de servicio que brindan las tecnologías de la información? & $\begin{array}{llllll}0 & 1 & 2 & 3 & 4 & 5 \\
\end{array}$ \\
\hline PRC4 & ¿Control económico de las tecnologías de la información? & $\begin{array}{llllll}0 & 1 & 2 & 3 & 4 & 5 \\
\end{array}$ \\
\hline PRC5 & $\begin{array}{l}\text { ¿Procesos estándares de mejores prácticas (COBIT, Objetivos de Control para la Información y la } \\
\text { Tecnología relacionada), como marco de trabajo para el Gobierno de TI? }\end{array}$ & 0122345 \\
\hline \multicolumn{3}{|c|}{ 3. Madurez del Gobierno de TI } \\
\hline MGO1 & ¿Madurez del Gobierno de TI? & $0 \begin{array}{llllll}0 & 1 & 2 & 3 & 4 & 5 \\
\end{array}$ \\
\hline
\end{tabular}

\section{Instrumento para colección de información}

Se ha diseñado un instrumento con 12 preguntas (indicadores), relacionadas a los constructos: estructuras, procesos, madurez del Gobierno de Tecnologías de la Información, como se indica en la Tabla 3. Es notorio que el instrumento proviene de la operacionalización de las variables.

\section{Resultados}

La encuesta fue aplicada el primer trimestre de 2016

\section{Proceso de la Información}

- Los datos de la encuesta efectuada vía web, se almacenan en estructuras de datos en formato Excel.

- Los datos en Excel son migrados a SPSS.

- El modelo estructural es implementado con el software Smart PLS 3.1.9

- La data en Smart PLS 3.1.9, se importa desde SPSS.

- Con el modelo generado en Smart PLS 3.1.9, se generan los estadísticos que corresponden a modelos estructurales, para dar validez al modelo.

Figura 3. Resultados del modelo estructural

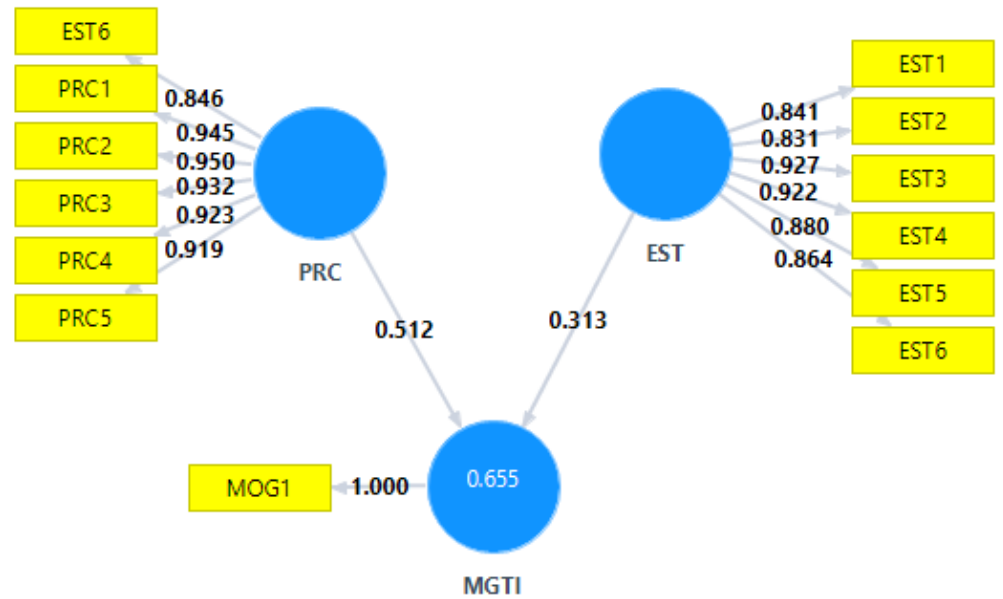


El modelo propuesto es sometido a análisis a través de dos fases:

Primero la validez y fiabilidad del modelo de medida.
En donde se determina, si los conceptos teóricos están medidos de manera correcta a través de las variables observadas. En la en la figura 3 se ilustran los resultados del modelo estructural y en la Tabla 4 se sintetiza esta evaluación.

Tabla 4. Fiabilidad del modelo de medida.

\begin{tabular}{|l|l|}
\hline \multicolumn{1}{|c|}{ Parámetros } & \multicolumn{1}{c|}{ Valores Obtenidos } \\
\hline $\begin{array}{l}\text { Fiabilidad individual del } \\
\text { ítem. }\end{array}$ & Todas las cargas están por encima de 0.7. Ver figura 3, valores entre constructos e indicadores. \\
\hline $\begin{array}{l}\text { Fiabilidad de cada } \\
\text { constructo }\end{array}$ & $\begin{array}{l}\text { Los valores para alfa de Cronbach de los constructos superan el valor 0.7, lo que da validez al } \\
\text { constructo. Como se indica en la Tabla 5. } \\
\text { En el análisis de fiabilidad compuesta, todos los constructos del modelo presentan valores } \\
\text { superiores a 0.6, confirmándose por tanto la consistencia interna de todos. Tabla 6. }\end{array}$ \\
\hline Validez convergente & $\begin{array}{l}\text { Para evaluar la validez convergente de los constructos se hace uso de la varianza extraída media } \\
\text { (AVE). Los valores para el indicador AVE, superan el valor mínimo recomendable de 0.5 Tabla 7. }\end{array}$ \\
\hline Validez discriminante & $\begin{array}{l}\text { Con el chequeo de cargas cruzadas, se valida que cada indicador tiene correlación con su propia } \\
\text { variable latente antes que con otras. Tabla 8 }\end{array}$ \\
\hline
\end{tabular}

\section{Fuente: El autor.}

Tabla 5. Fiabilidad del constructo

\begin{tabular}{|l|c|}
\hline & Cronbachs Alpha \\
\hline EST & 0.940 \\
\hline MGTI & 1.000 \\
\hline PRC & 0.963 \\
\hline
\end{tabular}

Tabla 6. Fiabilidad compuesta

\begin{tabular}{|l|c|}
\hline & Composite Reliability \\
\hline EST & 0.953 \\
\hline MGTI & 1.000 \\
\hline PRC & 0.970 \\
\hline
\end{tabular}

Tabla 7. Validez convergente

\begin{tabular}{|c|c|}
\hline & AVE \\
\hline EST & 0.771 \\
\hline MGTI & 1.000 \\
\hline PRC & 0.846 \\
\hline
\end{tabular}

En segundo lugar la valoración del modelo estructural, en base a la evaluación del peso y la magnitud de las relaciones entre las distintas variables.
Tabla. 8. Cargas cruzadas. Validez discriminante

\begin{tabular}{|l|r|l|l|}
\hline & \multicolumn{1}{|l|}{ EST } & MGTI & PRC \\
\hline EST1 & 0.841 & & \\
\hline EST2 & 0.831 & & \\
\hline EST3 & 0.927 & & \\
\hline EST4 & 0.922 & & \\
\hline EST5 & 0.880 & & \\
\hline EST6 & 0.864 & & \\
\hline MOG1 & & 1.000 & \\
\hline PRC1 & & & 0.945 \\
\hline PRC2 & & & 0.950 \\
\hline PRC3 & & & 0.932 \\
\hline PRC4 & & & 0.923 \\
\hline PRC5 & & & 0.919 \\
\hline
\end{tabular}

La Tabla 9, contiene los datos de esta evaluación, en ella constan: el índice $\mathrm{R}^{2}$, el efecto $f^{2}$, los coeficientes path estandarizados $\beta$, y el análisis de Bootstrapping. 
Tabla 9. Evaluación del modelo estructural.

\begin{tabular}{|l|l|}
\hline \multicolumn{1}{|c|}{ Parámetro } & \multicolumn{1}{c|}{ Valores obtenidos del modelo } \\
\hline Índice $R^{2}$ & $\begin{array}{l}\text { La medida del poder predictivo del modelo para las variables latentes dependientes, se } \\
\text { obtiene con el índice } \mathrm{R}^{2} \text {, son mayores a 0.1, lo que ratifica la característica predictiva } \\
\text { del modelo. Como se indica en la Tabla 10. }\end{array}$ \\
\hline Efecto $f^{2}$ & $\begin{array}{l}\text { Mide el impacto sobre un constructo dependiente de una variable latente, para el } \\
\text { modelo se presentan valores que se encuentran en el rango permisible. Como se indica } \\
\text { en la Tabla 11. }\end{array}$ \\
\hline $\begin{array}{l}\text { Coeficientes path } \\
\text { estandarizados } \beta\end{array}$ & $\begin{array}{l}\text { Para el modelo se supera el valor mínimo de 0.2, razón por la cual desde el punto de } \\
\text { vista estructural el modelo no debería ser reorganizado. Tabla 12. }\end{array}$ \\
\hline Análisis de Bootstrapping & $\begin{array}{l}\text { Bootstrap también determina el cálculo del error estándar de los parámetros y los } \\
\text { valores “t” de Student; en este ámbito se consideran como significativos a los } \\
\text { indicadores cuyo "t" de Student es mayor que 1,96. No todos los valores son } \\
\text { superiores a 1,96, como se indica en la Tabla 12. }\end{array}$ \\
\hline
\end{tabular}

Tabla $10 . R^{2}$ de las variables latentes dependientes.

\begin{tabular}{|l|l|}
\hline & R Square \\
\hline MGTI & 0.655 \\
\hline
\end{tabular}

Tabla 11. $f^{2}$ de las variables latentes dependientes

\begin{tabular}{|l|l|c|l|}
\hline & EST & MGTI & PRC \\
\hline EST & & 0.043 & \\
\hline MGTI & & & \\
\hline PRC & & 0.114 & \\
\hline
\end{tabular}

La tabla 12 despliega las relaciones entre los constructos del modelo a través de los caminos beta estandarizados, el error estándar, el valor de t de student, el nivel de significancia y la aceptación o rechazo de la hipótesis.

Tabla 12. Relaciones entre constructos

\begin{tabular}{|c|c|c|c|c|c|c|}
\hline $\begin{array}{l}\text { Hipótesis relaciones } \\
\text { entre constructos }\end{array}$ & $\boldsymbol{\beta}$ & $\begin{array}{l}\text { Error } \\
\text { estándar }\end{array}$ & t - student & $\begin{array}{c}\text { Valores } \\
\text { p }\end{array}$ & $\begin{array}{c}\text { Nivel de } \\
\text { significancia }\end{array}$ & $\begin{array}{l}\text { Aceptación o } \\
\text { rechazo }\end{array}$ \\
\hline EST -> MGTI & 0.313 & 0.115 & 2.724 & 0.007 & $* *$ & Aceptado \\
\hline PRC -> MGTI & 0.512 & 0.112 & 4.573 & 0.000 & $* * *$ & Aceptado \\
\hline
\end{tabular}

$* * * p<0.001 ; * * p<0.01 ; * p<0.05$ 


\section{Conclusiones}

Con respecto a las hipótesis uno y dos; se encontró que la uno manifiesta relación medianamente significativa entre las estructuras y la Madurez del Gobierno de TI con un valor beta de 0.313. La hipótesis dos, mantiene relación altamente significativa entre los procesos y la Madurez del Gobierno de TI, con un valor beta de 0.512 . Resultado que se apoya los hallazgos de Teo, Manaf y Fong Choong [26] en el sentido de que el Gobierno de TI requiere de estructuras y procesos adecuados para motivar iniciativas para su desarrollo, en donde el valor beta de los dos caminos es superior a 0.355.

Con la finalidad de entender ¿por qué? el constructo estructuras no es altamente significativo, se describe la posible razón: un porcentaje significativo de los encuestados tienen menos de cinco años laborando en la universidad y no han recibido o no cuentan con la inducción necesaria sobre las estructuras del Gobierno de TI. Resultados que validan lo indicado por Teo, Manaf y Fong [27] en su investigación sobre los usuarios de TI, en donde se determina que los bajos niveles de percepción sobre la prácticas de Gobierno de TI en la organización son resultado de la débil socialización y entrenamiento interno.

\section{Futuras investigaciones}

Para una instancia futura de investigación, se recomienda considerar la variable "mecanismos relacionales", como alternativa que propondrá una variación al modelo presentado en este trabajo.

\section{Agradecimientos}

A la Unidad Académica de Tecnología de la Información y la Comunicación, de la Universidad Católica de Cuenca, por la apertura a procesos de orden investigativo sobre aspectos de las Administración de Tecnologías de la Información.

\section{Referencias}

[1] D. Cordero, «Modelo para Gobierno de Tecnologías de la Información (GTI): caso de las Universidades Cofinanciadas de la Zona 6 de la República del Ecuador (Tesis de Grado Doctoral),» México, 2016.

[2] W. Grembergen y S. De Haes, Enterprise Governance of Information Technology, New York: Springer, 2009, p. 6

[3] R. Kingsford, L. Dunn y J. Cooper, «Information Systems, IT Governance and
Organisational Culture,» de 14th Australasian Conference on Information Systems, Perth, Western Australia, 2003.

[4] A. Brown y G. Grant, «Framing the Frameworks: A Review of IT Governance Research. Communications of the Association for Information Systems,» Communications of the Association for Information Systems, vol. 15, pp. 696-712, 2005.

[5] X. Zhong, S. Vatanasakdakul y C. Aoun, IT Governance in China: Cultural Fit and IT Governance Capabilities, Ho Chi Minh City, 2012.

[6] E. Nfuka y L. Rusu, «Critical success factors for effective IT governance in the public sector organizations in a developing country: the case of Tanzania,» de Proceedings of AMCIS 2010, Lima, 2010.

[7] G. Hofstede, Secon Edition. Culture's Consequences: Comparing Values, Behaviors, Institutions, and Organizations Across Nations., Unites States of America: Sage Publication Ltd, 2001, pp. 6-12.

[8] J. Luftman, «Assessing Business-IT alignment Maturity,» Communications of AIS, vol. 4, 2000.

[9] R. Kingsford y L. Dunn, «Information Systems, IT Governance and Organisational Culture,» 14th Australasian Conference on Information Systems Perth, 2003.

[10] C. Pollard y G. Ridley, «Attempting to Define IT Governance: Wisdom or Folly?,» Proceedings of the 39th Hawaii International Conference on System Sciences, p. Hawaii, 2006.

[11] R. Peterson, «Integration Strategies and Tactics for Information Technology Governance,» de Strategies for Information Technology Governance, Hershey, Idea Group Publishing, 2003, pp. 37-70.

[12] R. Peterson, «Crafting Information Technology Governance,» Information Systems Management, vol. 21, pp. 7-22, 2004.

[13] S. De Haes y W. Grembergen, «Information Technology Governance Best Practices in Belgian Organisations,» Proceedings of the 39th Hawaii International Conference on System Sciences, pp. 1-9, 2006.

[14] J. Luftman y T. Brier, «Achieving and Sustaining Business-IT alignment,» California Management Review, vol. 42, $\mathrm{n}^{\mathrm{o}}$ 1, pp. 109122, 1999.

[15] Ministry of Technology, Innovation and Citizens's Services, «Office of the Chief Information Officer,» 2014. [En línea]. 
Available: http://www.cio.gov.bc.ca/. [Último acceso: 5 Enero 2014].

[16] J. M. Earl, «Experiences in Strategic Information Systems Planning,» MIS Quarterly, vol. 17, n 1, pp. 1-24, Marzo 1993.

[17] W. Van Grembergen, R. Saull y S. De Haes, «Linking the IT Balanced Scorecard to the Business Objectives at a major Canadian Financial group,» Journal for Information Technology Cases and Applications (JITCA), vol. 5, $\mathrm{n}^{\circ}$ 1, pp. 1-27, 2003.

[18] A. Parisa, R. Lazar y H. Shengnan, «The Influence of Culture on IT Governance: A Literature Review,» 47th Hawaii International Conference on System Science, pp. 4436-4445, 2014.

[19] W. Van Grembergen, S. De Haes y I. Amelinckx, «Using CobiT and the Balanced Scorecard as Instruments for Service Level Management,» Information Systems Control Journal, vol. 4, pp. 1-7, 2003.

[20] M. Parker, R. Benson y H. Trainor, Information Economics: Linking Business Performance to Information Technology, USA: Prentice Hall, 1988.

[21] J. C. Henderson, N. Venkatraman y S. Oldach, «Continuous Strategic Alignment, Exploiting Information Technology Capabilities for Competitive Success,» European Management Journal, vol. 11, n 2, p. 139-149, 1993.
[22] C. Fornell y D. F. Larcker, «Evaluating Structural Equation Models with Unobservable Variables and Measurement Error,» Journal of Marketing Research, vol. 18, ${ }^{\circ}$ 1, pp. 39-50, February 1981.

[23] J. J. Céspedes Lorente y M. Sánchez Pérez, «Tendencias y desarrollos recientes en métodos de investigación y análisis de datos en dirección de empresas,» Revista Europea de Dirección y Economía de la Empresa, vol. 5, no 3, pp. 23-40, 1996.

[24] G. Cepeda y J. L. Roldán, «Aplicando en la práctica la técnica PLS en la Administración de Empresas,» Facultad de Ciencias Económicas y Empresariales, Sevilla, 2004.

[25] A. J. Caballero Domínguez, «SEM vs. PLS: Un Enfoque Basado en la Práctica,»IV Congreso de Metodología de Encuestas., pp. 57-66, 2022 Septiembre 2006.

[26] W. Teo, A. Manaf y P. Fong Choong, «Information Technology Governance: Applying the Theory of Planned Behaviour,» Journal of Organizational Management Studies, vol. 2013, pp. 1-15, 2013.

[27] W. Teo, A. Manaf y P. Fong, «Perceived Effectiveness of Information Technology Governance Initiatives Among IT Practitioners,» International Journal of Engineering Business Management, vol. 5, $\mathrm{n}^{\circ}$ 19, pp. 1-9, 2013. 